\title{
COMPLETELY DISTRIBUTIVE CSL ALGEBRAS WITH NO COMPLEMENTS IN $\mathcal{C}_{p}$
}

\author{
J. A. ERDOS
}

(Communicated by Palle E. T. Jorgensen)

\begin{abstract}
Anoussis and Katsoulis have obtained a criterion for the space $\operatorname{Alg} \mathcal{L} \cap \mathcal{C}_{p}$ to have a closed complement in $\mathcal{C}_{p}$, where $\mathcal{L}$ is a completely distributive commutative subspace lattice. They show that, for a given $\mathcal{L}$, the set of $p$ for which this complement exists forms an interval whose endpoints are harmonic conjugates. Also, they establish the existence of a lattice $\mathcal{L}$ for which $\operatorname{Alg} \mathcal{L} \cap \mathcal{C}_{p}$ has no complement for any $p \neq 2$. However, they give no specific example. In this note an elementary demonstration of a simple example of this phenomenon is given. From this it follows that for a wide range of lattices $\mathcal{L}$, Alg $\mathcal{L} \cap \mathcal{C}_{p}$ fails to have a complement for any $p \neq 2$.
\end{abstract}

Let $\mathcal{L}$ be a completely distributive commutative subspace lattice. In [AN] the authors obtain a criterion for the space $\operatorname{Alg} \mathcal{L} \cap \mathcal{C}_{p}$ to have a closed complement in the von Neumann-Schatten class $\mathcal{C}_{p}$ and show that, for a given $\mathcal{L}$, the set of $p$ for which this complement exists forms an interval whose endpoints are harmonic conjugates. The well-known triangular integral procedure shows that in the case of a nest this interval is $(1, \infty)$, while if $\mathcal{L}$ is the Boolean algebra generated by the orthogonal projections onto the elements of an orthonormal basis, then the interval is $[1, \infty]$. In [AN], Corollary 5 , the authors prove that there exists a lattice $\mathcal{L}$ for which $\operatorname{Alg} \mathcal{L} \cap \mathcal{C}_{p}$ has no complement for any $p \neq 2$. However, there is no example given since the demonstration is purely an existence proof based on non-convergence of a certain net and uses a sophisticated example of Froelich [F].

In this note we use known techniques to exhibit a simple specific algebra which does not have a complement in $\mathcal{C}_{p}$ for $p \neq 2$. Using a result of Davidson and Power [DP] it will follow that if $\mathcal{L}$ is any infinite tensor product of non-trivial nests, then Alg $\mathcal{L} \cap \mathcal{C}_{p}$ fails to have a closed complement in $\mathcal{C}_{p}$ for $p \neq 2$.

Example (The discrete Sierpinski algebra). The discrete Sierpinski algebra is defined to be the set of all bounded operators on $l^{2}$ whose matrix representations with respect to the standard basis have entries of 0 at all points not designated by $*$ in Figure 1.

Received by the editors October 3, 1994

1991 Mathematics Subject Classification. Primary 47D25; Secondary 47B10.

Key words and phrases. Commutative subspace lattice, complemented subspace, von Neumann-Schatten class. 


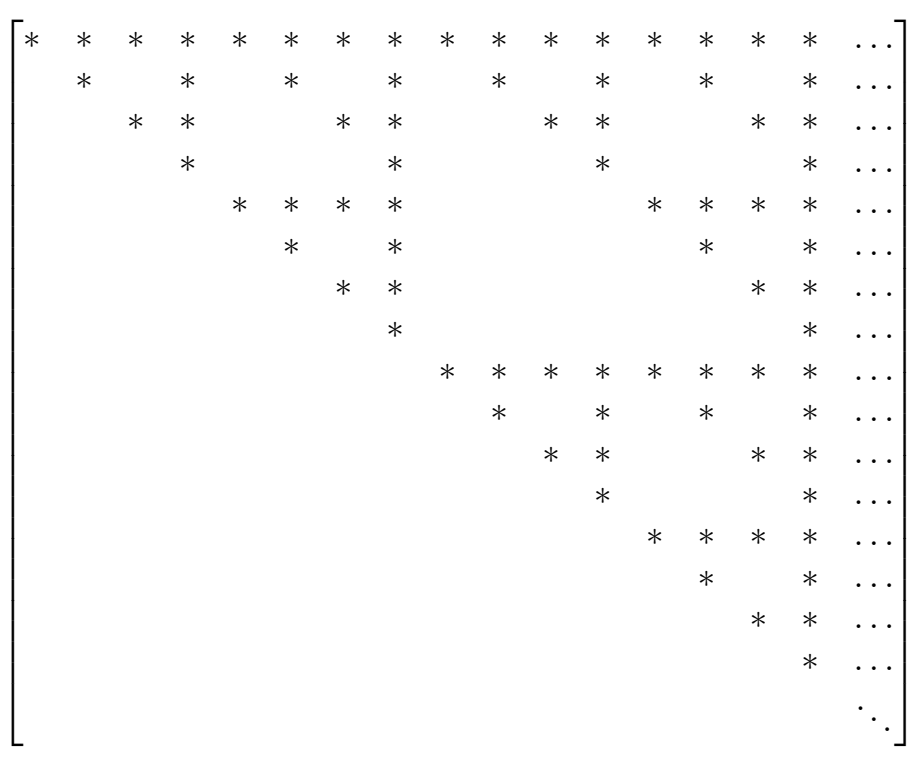

FIGURE 1

This algebra is (isomorphic to) $\operatorname{Alg} \mathcal{L}$ where $\mathcal{L}$ is the tensor product of an infinite number of copies of $\mathcal{N}_{2}$, the obvious non-trivial nest on $l_{2}^{2}$.

Claim. Let $\mathcal{A}$ be the Sierpinski algebra. Then $\mathcal{A} \cap \mathcal{C}_{p}$ has no complement in $\mathcal{C}_{p}$ for $p \neq 2$.

To establish the claim, we first quote the following result of Anoussis and Katsoulis.

Theorem $\left(\left[\mathrm{AN}\right.\right.$, Theorem 1]). Let $\mathcal{L}$ be a $C S L$, let $\mathcal{D}=\mathcal{L}^{\prime \prime}$ be the von Neumann algebra generated by $\mathcal{L}$ and let $1<p<\infty$. Then $\operatorname{Alg} \mathcal{L} \cap \mathcal{C}_{p}$ has a complement in $\mathcal{C}_{p}$ if and only if there is a bounded idempotent $\pi$ from $\mathcal{C}_{p}$ onto $\operatorname{Alg} \mathcal{L} \cap \mathcal{C}_{p}$ such that

$$
\pi(A X B)=A \pi(X) B
$$

for all $X$ in $\mathcal{C}_{p}$ and $A, B$ in $\mathcal{D}$.

Note that for the Sierpinski algebra $\mathcal{A}, \mathcal{D}$ is the set of all diagonal matrices.

Suppose that an idempotent $\pi$ of the above form exists in the case when $p \neq 2$. Since the set of $p$ for which such a projection exists is an interval whose endpoints are harmonic conjugates, we may assume that $p<2$. We show that this leads to a contradiction.

Observation 1. The idempotent $\pi$ is formally Schur multiplication by the matrix $S$ in Figure 2 (the unspecified entries of the matrix $S$ are 0 ). This certainly holds for $\pi(X)$ when the matrix of $X$ has all but a finite number of its entries equal to zero. 


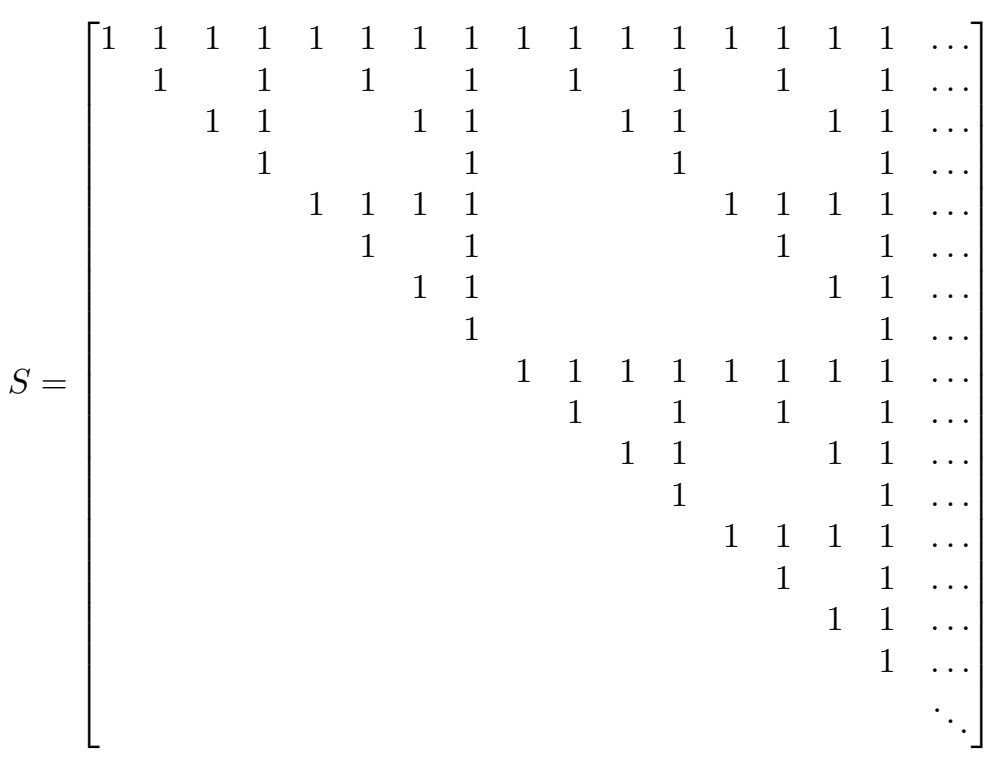

FIGURE 2

This is clear since if $\delta_{i j}$ are the standard matrix units, $\delta_{i j} \in \mathcal{A}$ if and only if the $(i, j)$ th entry of $S$ is 1 . Thus, writing $E_{i}(\in \mathcal{D})$ for the projection onto the $i$ th basis element,

$$
E_{i} \pi(X) E_{j}=\pi\left(E_{i} X E_{j}\right)=\pi\left(X_{i j}\right)=S_{i j} X_{i j} \delta_{i j}
$$

and the observation follows by a simple summation.

Observation 2. It is sufficient to exhibit finite matrices with entries all either 0 or 1 whose action of Schur multiplication on $\mathcal{C}_{p}$ has arbitrarily large norm.

Note that any finite matrix whose entries are all either 0 or 1 is a submatrix of $S$. Indeed, the $\left(2^{k}+1\right)$ th rows of $S$ for $k=0,1, \ldots, r-1$ are:

$$
\begin{aligned}
& \begin{array}{lllllllllllllllll}
0 & 1 & 0 & 1 & 0 & 1 & 0 & 1 & 0 & 1 & 0 & 1 & 0 & 1 & 0 & 1 \ldots
\end{array} \\
& \begin{array}{llllllllllllllll}
0 & 0 & 1 & 1 & 0 & 0 & 1 & 1 & 0 & 0 & 1 & 1 & 0 & 0 & 1 & 1 \ldots
\end{array} \\
& \begin{array}{llllllllllllllll}
0 & 0 & 0 & 0 & 1 & 1 & 1 & 1 & 0 & 0 & 0 & 0 & 1 & 1 & 1 & 1 \ldots
\end{array} \\
& \text { : } \vdots \quad \vdots \quad \text { : }
\end{aligned}
$$

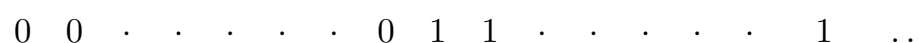

and these contain all possible columns of length $r$. Now, if $E$ and $F$ are respectively the projections of $l^{2}$ onto the span of the basis elements corresponding to the rows and columns of a submatrix $M$ of $S$, it is clear from Observation 1 and the relation

$$
E \pi(X) F=\pi(E X F)
$$

that Schur multiplication by $M$ has the same norm as the map $X \mapsto E \pi(X) F$ and this is at most $\|\pi\|$.

We now exhibit the required matrices. Define the $2^{n} \times 2^{n}$ matrices $U_{n}$ by

$$
U_{1}=\left(\begin{array}{cc}
1 & 1 \\
-1 & 1
\end{array}\right), \quad U_{n+1}=U_{n} \otimes U_{1}
$$


Let $H_{n}$ be the $2^{n} \times 2^{n}$ matrix with every entry equal to 1 . We estimate the norm of $\mathcal{P}_{n}$, the operator of Schur multiplication by $P_{n}=\frac{1}{2}\left(U_{n}+H_{n}\right)$. Note that

$$
P_{n}=\left[\begin{array}{ccccccccc}
1 & 1 & 1 & 1 & 1 & 1 & 1 & 1 & \ldots \\
0 & 1 & 0 & 1 & 0 & 1 & 0 & 1 & \ldots \\
0 & 0 & 1 & 1 & 0 & 0 & 1 & 1 & \ldots \\
1 & 0 & 0 & 1 & 1 & 0 & 0 & 1 & \ldots \\
0 & 0 & 0 & 0 & 1 & 1 & 1 & 1 & \ldots \\
1 & 0 & 1 & 0 & 0 & 1 & 0 & 1 & \ldots \\
1 & 1 & 0 & 0 & 0 & 0 & 1 & 1 & \ldots \\
0 & 1 & 1 & 0 & 1 & 0 & 0 & 1 & \ldots \\
\vdots & \vdots & \vdots & \vdots & \vdots & \vdots & \vdots & \vdots & \ddots
\end{array}\right] .
$$

Since $\mathcal{P}_{n}\left(H_{n}\right)=P_{n}=\frac{1}{2}\left(U_{n}+H_{n}\right)$, it follows easily that the norm $\left\|\mathcal{P}_{n}\right\|_{p}$ of $\mathcal{P}_{n}$ as an operator on $\mathcal{C}_{p}$ satisfies

$$
2\left\|\mathcal{P}_{n}\right\|_{p} \geq \frac{\left\|U_{n}\right\|_{p}}{\left\|H_{n}\right\|_{p}}-1 .
$$

Since $2^{-n / 2} U_{n}$ is unitary, we have that

$$
\left\|2^{-n / 2} U_{n}\right\|_{p}^{p}=2^{n} .
$$

Also,

$$
H_{n}=(1,1, \ldots, 1)^{T} \otimes(1,1, \ldots, 1)
$$

has rank one and so

$$
\left\|H_{n}\right\|_{p}=\left\|H_{n}\right\|_{\infty}=\|(1,1, \ldots, 1)\|^{2}=2^{n} .
$$

Therefore,

$$
\frac{\left\|U_{n}\right\|_{p}}{\left\|H_{n}\right\|_{p}}=2^{n(1 / p-1 / 2)} \rightarrow \infty \quad \text { if } p<2 .
$$

The claim now follows.

Corollary. For any lattice $\mathcal{L}$ that is the infinite tensor product of non-trivial nests, $\operatorname{Alg} \mathcal{L} \cap \mathcal{C}_{p}$ has no closed complement in $\mathcal{C}_{p}$ for $p \neq 2$.

This is immediate from Lemma 2.1 of [DP] which shows that any matrix with all entries 0 or 1 can be embedded in $\operatorname{Alg} \mathcal{L}$.

Remarks. 1. Note that the lack of a uniform bound for $\left\|\mathcal{P}_{n}\right\|$ and the above result of Davidson and Power may be used to prove the general result without reference to the Sierpinski algebra. However, the elementary nature of the example and proof are of sufficient interest to justify their inclusion.

2. Anoussis and Katsoulis [AN] show that the set of $p$ such that the space Alg $\mathcal{L} \cap \mathcal{C}_{p}$ has a closed complement in $\mathcal{C}_{p}$ forms an interval whose endpoints are harmonic conjugates. It is an open question whether this interval can ever have its lower endpoint strictly between 1 and 2 .

3. A weaker form of the question posed in Remark 2 has been solved by Olevskii and Solomyak [OS]. They show that there exist infinite matrices $A$ (not with entries 
constrained to be 0 or 1 ) such that the set of $p$ for which the operation of Schur multiplication by $A$ is bound $\left(\mathcal{C}_{p} \rightarrow \mathcal{C}_{p}\right)$ is a proper harmonic interval with finite endpoints.

I wish to thank Brian Davies for some helpful conversations concerning this work.

\section{REFERENCES}

[AN] M. Anoussis and E. G. Katsoulis, Complemented subspaces of $\mathcal{C}_{p}$ spaces and CSL algebras, J. London Math. Soc. (2) 45 (1992), 301-313. MR 93i:47064

[DP] K. R. Davidson and S. C. Power, Failure of the distance formula, J. London Math. Soc. (2) 32 (1984), 157-165. MR 87e:47056

[F] J. Froelich, Compact operators, invariant subspaces and spectral synthesis, J. Funct. Anal. 81 (1988), 1-37. MR 90b: 47078

[OS] V. Olevskii and M. Solomyak, An estimate for Schur multipliers in $\mathcal{S}_{p}$ classes, Linear Algebra Appl. MR 95f: 47050

Department of Mathematics, King's College, London WC2R 2LS, United Kingdom E-mail address: J.ERDOS@uk.ac.kcl 